Check for updates

Cite this: RSC Adv., 2017, 7, 39403

\title{
Study of the estrogenic-like mechanism of glycosides of cistanche using metabolomics $\uparrow$
}

\author{
H. Song, ${ }^{\text {ac }}$ W. L. Li, ${ }^{\star b}$ B. M. Liu, ${ }^{d}$ X. M. Sun, ${ }^{a}$ J. X. Ding, ${ }^{a}$ N. Chen, ${ }^{a}$ Y. B. Jiac \\ and Z. Xiang (D) *a
}

Cistanche deserticola, known as Rou Cong-Rong in China, has been used as a tonic for more than 1800 years, with previous studies demonstrating that glycosides of cistanche (GCs) are a main active component. In this study, a uterotrophic assay and histological analysis were utilized to confirm the estrogenic activity of GCs, and UPLC-MS/MS-based metabolomics was used to explore the estrogeniclike mechanism of GCs in serum and urine. Seven altered differential metabolites, including citric acid, taurine, proline, betaine, ornithine, pyroglutamic acid, and $\alpha$-ketoglutaric acid, were of particular interest due to being present in both serum and urine. Moreover, the differential metabolites were categorized into several major pathways, including the citrate cycle (TCA cycle), glutathione metabolism, arginine and proline metabolism, D-glutamine and D-glutamate metabolism, phenylalanine, tyrosine, and tryptophan biosynthesis, and phenylalanine metabolism. The estrogenic-like mechanism of GCs could be concluded as closely related to the TCA cycle and glutathione metabolism due to these pathways being present in both serum and urine. Our results shed light on the estrogenic-like mechanism of GCs, which will be helpful for GC development and utilization.

Received 21st June 2017

Accepted 1st August 2017

DOI: $10.1039 / \mathrm{c} 7 \mathrm{ra06930h}$

rsc.li/rsc-advances and fertility functions, and has been used as a tonic for more than 1800 years. $^{8,9}$ Recently, pharmacology studies have demonstrated that this tonic has broad medicinal functions, such as hormone regulation, aperient, immunomodulatory, anti-oxidative, anti-apoptotic, neuroprotective, antinociceptive, anti-inflammatory, anti-fatigue, and estrogenic activities. ${ }^{10}$ Glycosides of cistanche (GCs) extracted from CD are among the main active components and exhibit various biological activities. ${ }^{11}$ Although the active constituents of $\mathrm{CD}$ have been elucidated previously, ${ }^{12}$ the estrogenic-like mechanism of GCs has never been investigated.

In this continuing study, we aimed to confirm the possible use of GCs as phytoestrogens and carry out a metabolomics analysis to explore the estrogenic-like mechanism of GCs. First, a uterotrophic assay and histological analysis were utilized to confirm the estrogenic activity of GCs. Most importantly, we focused on the metabolic changes in rat serum and urine using UPLC-MS/MS-based metabolomics analysis. Due to the deficiencies of non-targeted metabolomics, such as repeatability and complicated matrix \footnotetext{
influence, an MRM mode-based pseudo method was used to specifically monitor the metabolites, and indexes (including energy metabolism, oxidative stress, lipid metabolism, and amino metabolism) related to estrogenic effects, growth, and development were selected as biomarkers for detection. Our results shed light on the estrogenic-like mechanism of GCs, which will aid the development and utilization of GCs.

${ }^{a}$ Institute of Materia Medica, Research Center of Life Sciences and Environmental Sciences, Harbin University of Commerce, Harbin, Heilongjiang 150076, China. E-mail: rainbowaftersnow@hotmail.com

${ }^{b}$ School of Pharmacy, Harbin University of Commerce, Harbin, Heilongjiang 150076, China.E-mail: lwldzd@163.com

${ }^{c}$ Engineering Research Center of Natural Anticancer Drugs of Ministry of Education, Harbin University of Commerce, Harbin, Heilongjiang 150076, China

${ }^{d}$ Heilongjiang Provincial Hospital, Harbin, Heilongjiang 150001, China

$\dagger$ Electronic supplementary information (ESI) available. See DOI:
} 10.1039/c7ra06930h 


\section{Experimental}

\section{Reagents}

L-Leucine, L-kynurenine, L-tryptophan, 5-HTP, cholic acid, $N$ phenylacetylglycine, 5-HT, glutathione (GSH), and 2,4-dinitrophenylhydrazine ( $\geq 99.0 \%$, HPLC) were purchased from Dalian Melone Biology Technology Co. (Dalian, China). $N$-Ethylmaleimide ( $\geq 98 \%$, HPLC), L-glutathione oxidized (GSSG, $\geq 98 \%$, HPLC), and 1,1,3,3-tetraethoxypropane (TEP) were purchased from Sigma (Madrid, Spain). L-Phenylalanine (Ring-D5, 98\%, DLM-1258-5) was purchased from Cambridge isotope laboratories (MA, USA). MS-grade methanol and acetonitrile were purchased from ACS (Houston, USA). Diethylstilbestrol (purity, $\geq 99.0 \%$ ), formic acid, glacial acetic acid, and hematoxylin and eosin (H\&E) were purchased from Sigma-Aldrich (Sigma Chemical Co., St. Louis, USA). Naringenin (purity, $\geq 98 \%$ (HPLC)) was purchased from Shanghai Jingchun Aladdin Reagent Co. (Shanghai, China). GCs were prepared in our laboratory, and their purity was determined to be $60 \%$ by ultraviolet spectrophotometry using acteoside as a marker for determination.

\section{Preparation of GCs}

Briefly, cistanche powder (100 g) was soaked in 75\% ethanol $(1000 \mathrm{~mL})$ for $1 \mathrm{~h}$, then reflux extracted for $2.5 \mathrm{~h}$ three times. The supernatant was concentrated under vacuum to obtain cistanche extract $(23.3 \mathrm{~g})$. The extract was diluted with water to a concentration of $0.5 \mathrm{~g} \mathrm{~mL}^{-1}$ (solubility determined with crude drug). After adsorption with AB-8 macroporous resin for $8 \mathrm{~h}$, the column was eluted sequentially with water and $85 \%$ ethanol. The $85 \%$ ethanol eluant was concentrated to obtain ethanol extract $(8.4 \mathrm{~g})$. Ethanol extract $(0.02 \mathrm{~g})$ was accurately weighed and dissolved in $50 \%$ methanol $(10 \mathrm{~mL})$. A $1 \mathrm{~mL}$ aliquot of the solution was diluted to $100 \mathrm{~mL}$ with methanol in a volumetric flask. Finally, the diluted solution was measured at $330 \mathrm{~nm}$ by ultraviolet spectrophotometry. The ultraviolet absorption was 0.341 , the linear relationship for acteoside by UV was $y=$ $24.905 X+0.0426\left(R^{2}=0.9982\right)$. When containing $5.04 \mathrm{~g}$ of GCs, the purification rate was $60 \%$ (acteoside was used as a marker for GC determination). Fingerprint evaluation of the GCs is shown in ESI Fig. S1. $\dagger$

\section{Animal experiments and sample collection}

Female SD rats of sexual immaturity (45-60 g) and sexual maturity (320-380 g) were provided by the Animal Center of Harbin Medical University, laboratory animal license, SCXK(Army): 2013-001. The animals were housed under SPF laboratory conditions and provided with a standard laboratory diet and filtered tap water ad libitum. All animal procedures were approved by the Heilongjiang Provincial Animal Welfare and Care Guidelines, and performed according to the National Institute of Health guidelines regarding the principles of animal care (2004). All rats used in this experiment were acclimatized to the above environment for a week.

The sexually immature SD rats were randomly divided into three groups of 10 rats: blank group, diethylstilbestrol group, and GC group. Meanwhile, 10 SD rats of sexual maturity were selected as the control group. The diethylstilbestrol group was i.g administered with diethylstilbestrol $\left(0.35 \mathrm{mg} \mathrm{kg}^{-1}, 1 \mathrm{~mL} /\right.$ $100 \mathrm{~g}$ ), the GC group was i.g administered with the GC solution (30 $\mathrm{g} \mathrm{kg}^{-1}, 1 \mathrm{~mL} / 100 \mathrm{~g}$ ), and the blank group and control group received distilled water of the same volume twice a day (morning and evening) for 3 days. On the third day, the rats were housed in metabolic cages after the administration was finished and urine samples were continuously collected for $24 \mathrm{~h}$. The rats were anesthetized using pentobarbital and blood samples were obtained and collected from abdominal aorta and centrifuged at $3000 \times g\left(15 \mathrm{~min}, 4{ }^{\circ} \mathrm{C}\right)$ to obtain serum. All samples were stored at $-20{ }^{\circ} \mathrm{C}$. Furthermore, the uterus was separated, weighed, and fixed using $10 \%$ formalin.

\section{Histological analysis}

Serial $5 \mu \mathrm{m}$-thick tissues were cut from the fixed uterus. The tissue sections were then embedded in paraffin, stained with H\&E using routine methods, and observed with an optical microscope (BZ-9000; Keyence, Osaka, Japan). The height of uterine epithelial cells was measured with a micrometer under the microscope.

\section{Metabolomics}

Preparation of activated charcoal stripped serum. Special blank serum was prepared from rat serum that had been stripped of endogenous materials using activated charcoal powder. Activated charcoal powder ( $6 \mathrm{~g}$ ) was added to the rat serum $(100 \mathrm{~mL})$ and shaken for $2 \mathrm{~h}$ at room temperature, and centrifuged at $4{ }^{\circ} \mathrm{C}$ and $13500 \mathrm{rpm}$ for $20 \mathrm{~min}$. The supernatant was filtered using Millipore express PES membranes (Merck Millipore, Ltd.) attached to a $20 \mathrm{~mL}$ syringe in the following sequence: $5 \mu \mathrm{m}, 1.2 \mu \mathrm{m}$, and $0.45 \mu \mathrm{m}$. The "stripped" serum was confirmed to be free of biomarkers by LC-MS/MS. ${ }^{\mathbf{1 3 , 1 4}}$

Preparation of standard solutions. L-Tryptophan (Try), L-kynurenine (Kyn), GSSG, $N$-phenylacetylglycine ( $N$-Phe), 5HTP, L-leucine (Leu), 5-HT, and cholic acid (CA) stock solutions $\left(1 \mathrm{mg} \mathrm{mL}{ }^{-1}\right.$ ) were prepared using $100 \%$ methanol. GSH was prepared at $0.5 \mathrm{mg} \mathrm{mL}^{-1}$ in $10 \mathrm{mM}$ NEM PBS buffer and stored at $-40{ }^{\circ} \mathrm{C}$ in brown bottles. Calibrators were generated using combined L-tryptophan, L-kynurenine, GSSG, $N$-phenylacetylglycine, 5-HTP, L-leucine, 5-HT, cholic acid, and GSH-NEM that were serially diluted with distilled water. The IS stock solution of $\mathrm{L}$-phenylalanine-d5 (d5-Phe) was prepared in 100\% methanol, and the working solution of $\mathrm{d} 5$-Phe $\left(10 \mathrm{ng} \mathrm{mL} \mathrm{mas}^{-1}\right)$ was prepared in $100 \%$ methanol and stored at $-40{ }^{\circ} \mathrm{C}$.

During the analysis, a derivatization step was necessary to avoid GSH degradation, which improved the stability for detection and quantification of GSH. GSH was determined after reaction with NEM..$^{15,16}$ According to the previous report, $50 \mathrm{mM}$ NEM was selected for GSH.

Sample preparation. For serum samples, $10 \mathrm{mM}$ NEM $(200 \mu \mathrm{L})$ was added to the sample $(200 \mu \mathrm{L})$, followed by methanol $\left(1000 \mu \mathrm{L}\right.$ containing IS Phe-d5 at $\left.10 \mathrm{ng} \mathrm{mL}^{-1}\right)$, and incubated for $20 \mathrm{~min}$ at $-20{ }^{\circ} \mathrm{C}$. After centrifugation at $4{ }^{\circ} \mathrm{C}$ and $12000 \mathrm{rpm}$ for $10 \mathrm{~min}$, the supernatants $(1000 \mu \mathrm{L})$ were transferred to $2 \mathrm{~mL}$ centritubes and evaporated to dryness. The dried 
residue was reconstituted in distilled water $(100 \mu \mathrm{L})$ after centrifugation for $15 \mathrm{~min}$ at $4{ }^{\circ} \mathrm{C}$ and $13500 \mathrm{rpm}$, and a $10 \mu \mathrm{L}$ aliquot of the supernatant was injected for analysis.

For urinary samples, $100 \mu \mathrm{L}$ of sample was placed in a $2 \mathrm{~mL}$ tube, and 1 volume of PBS (m/v) containing $50 \mathrm{mM}$ NEM was added to each urinary sample. Then, methanol $(1000 \mu \mathrm{L}$ containing IS at $10 \mathrm{ng} \mathrm{mL}^{-1}$ ) was added, and the sample was incubated at $-20{ }^{\circ} \mathrm{C}$ for $20 \mathrm{~min}$, and then centrifuged at $12000 \mathrm{rpm}$ for $10 \mathrm{~min}$ at $4{ }^{\circ} \mathrm{C}$. The supernatant $(1000 \mu \mathrm{L})$ was evaporated to dryness under a gentle stream of nitrogen at room temperature, and then the residue was dissolved in $60 \mu \mathrm{L}$ of mobile phase and vortexed for $1 \mathrm{~min}$ before centrifugation at $13500 \mathrm{rpm}$ and $4{ }^{\circ} \mathrm{C}$ for $15 \mathrm{~min}$. A $10 \mu \mathrm{L}$ aliquot supernatant was injected for analysis.

Methodology validation. Assay validation was performed according to the "Guidance for Industry: Bioanalytical Method Validation” (Food and Drug Administration, September 2013). The calibrators were generated using a surrogate matrix for Trp, Kyn, GSH, GSSG, 5-HT, 5-HTP, $N$-Phe, and CA from standard working solutions in the "stripped" serum. Each concentration calibrator was duplicated. The standard curve was calculated by $1 / X$ weighted least squares linear regression of standard curve calibrator concentrations and the peak area ratios for analyte to IS.

Precision and accuracy assay. The assay precisions of pooled QC samples and non-treated rat serum/urine QC samples were evaluated using one intra-day and three inter-day analytical runs. The QC samples with three different concentrations were generated by adding L-tryptophan, L-kynurenine, GSSG, $N$-phenylacetylglycine, 5-HTP, L-leucine, 5-HT, cholic acid, and GSH-NEM standard solutions to "stripped" serum/PBS, which were then processed in the same way as in vivo samples. Try, Kyn, GSSG, $N$-Phe, 5-HTP, 5-HT, Leu, and CA standard stock solutions ( $1 \mathrm{mg} \mathrm{mL}^{-1}$ ) were prepared in $100 \%$ methanol, except that of GSH, which was prepared at $0.5 \mathrm{mg} \mathrm{mL}^{-1}$ in $10 \mathrm{mM}$ NEM PBS buffer. Standard curves at eight different concentrations were generated in the same way as QC samples, and analyzed by $1 / X$ weighted least squares linear regression. For the method validation study, another QC method was performed simultaneously using pooled serum/urine $(50 \mu \mathrm{L})$ from each sample of the control or model groups to obtain a QC specimen, and then processed as in vivo samples. A series of QC samples and standard curves were run for every 50 animal samples.

Matrix effects. Matrix variation, defined as the variation in assay accuracy for different lots of matrix, was evaluated using six different plots of spiked rat serum/urine for analysis and assessing the spiked recovery. ${ }^{\mathbf{1 7}}$ Evaluation of the entire matrix effect for an endogenous compound assay is challenging. One way to test whether an assay has any (overall) matrix effect is to evaluate the spiked recovery. The spiked recovery test involves spiking the analyte into pre-extraction samples, which is different from the matrix factors (MF) test, which involves spiking analyte into post-extraction samples. ${ }^{18}$ The MF was calculated as the peak area of the analyte in the presence of biological matrix compared to that in the absence of biological matrix. The IS-normalized MF was used: $\mathrm{MF}=$ peak area/IS ratio in the presence of matrix $v s$. peak area/IS ratio in the absence of matrix. ${ }^{19}$
UPLC-MS/MS analysis. MS equipment equipped with a Waters X BridgeTM BEH C18 analytical column XP $(2.5 \mu \mathrm{m}$, $3.0 \times 100 \mathrm{~mm}$; Waters, Torrance, CA) was used for LC-MS/MS analysis. $Q$ linear gradient mobile phase composed of $0.1 \%$ formic acid water (solvent A) and methanol (solvent B) was used according to the following gradient program: $0-3.0 \mathrm{~min}(0-1 \%$ B); $3.0-10.0 \min (1-3 \% \quad \mathrm{~B}) ; \quad 10.0-14.0 \min (3-50 \% \quad \mathrm{~B})$; 14.0-18.0 $\min (50-95 \% \mathrm{~B}) ; 18.0-22.0 \mathrm{~min}$ (95-0\% B), the stop time is $25 \mathrm{~min}$. The injection volume was $10 \mathrm{~mL}$ and flow rate was $0.6 \mathrm{~mL} \min ^{-1}$. The final method used the following parameters: scan type, MRM; ion mode, electrospray ionization (ESI) in positive and negative ion modes; ion spray voltage, $\pm 4500 \mathrm{~V}$; curtain gas, 20 ; temperature, $450{ }^{\circ} \mathrm{C}$; ion source gas 1 , 40 ; ion source gas 2, 40. All UPLC-MS data were obtained using $\mathrm{AB}$ Analyst software (version 1.6.2), and the $m / z$ 171.1-125.2 for internal standard d5-Phe (DP, 63 V; CE, 8 V; CXP, 21 V; EP, 24 V) and detailed MS conditions are shown in the Table $\mathrm{S} 1 . \dagger$

Statistical analysis. All values were expressed as mean \pm SD. The significance of differences among groups was compared through one-way ANOVA test followed by Dunnett's test using the Statistical Package for Social Sciences program (SPSS 20.0, Chicago, IL, USA). The significance threshold was set at $p<0.05$ in this test.

\section{Results and discussion}

\section{Effect of GCs on the uterus}

Uterine weight and histological analysis were used to evaluate the effect of GCs on the uterus. Uterine weight was significantly increased in the diethylstilbestrol, GC, and control groups relative to the blank group $(P<0.01)$. In the blank group, the endometrial epithelium was low columnar with small glands, a cubic-shaped glandular epithelium, and without interstitial inflammatory cells. In the diethylstilbestrol group, the glandular epithelium and endometrium were high columnar and showed hyperplasia, with a lot of interstitial inflammatory cells. In the GC group, the glandular epithelium and endometrium were high columnar serrated and showed hyperplasia with a lot of interstitial inflammatory cells and intimal thickening. In the control group, the endometrium was high columnar and the glandular epithelium was low columnar, with few interstitial inflammatory cells. Compared with the blank group, the height of the uterine epithelial cells was significantly increased in the other groups $(P<0.01)$ (Fig. 1).

\section{Method validation of detected biomarker by UPLC-MS/MS}

Linearity and calibration range. For each measurement, a linear calibration curve with a weighting factor of $1 / X$ in the studied matrix was constructed. The calibration curves for analyses in serum were analyzed on different days. The calibration ranges for the analyses were distributed along seven calibration points, as shown in Table 1.

Accuracy and precision. The method was proven to be appropriate, in terms of accuracy and precision, both inter-day and intra-day, for all studied samples. The values of these parameters were less than $5 \%$ at the set concentrations for GSH, 

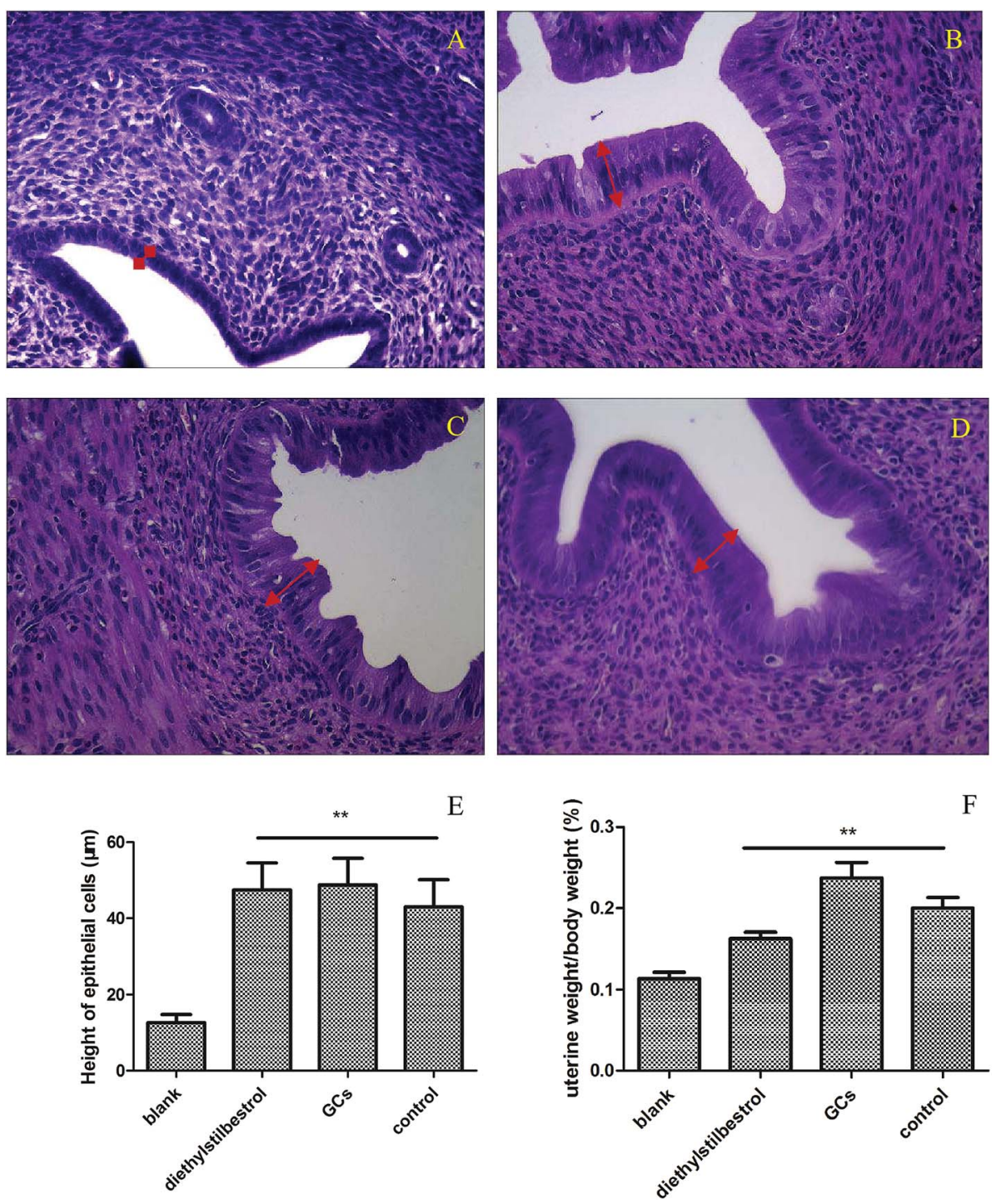

Fig. 1 Effect of GCs on uterine cells. Histological analysis (400× magnification) described as follows: (A) blank group; (B) diethylstilbestrol group; (C) GC group; (D) control group; (E) comparison of heights of uterine epithelial cells with the blank group $(* p<0.05, * * p<0.01)$; (F) comparison of uterine weight with the blank group $(* p<0.05, * * p<0.01)$.

GSSG, Leu, Trp, Kyn, 5-HTP, cholic acid, 5-HT, and N-phenylacetylglycine, with three replicates of each concentration analyzed. The data corresponding to the inter-day and intra-day values of accuracy and precision are shown in ESI Table S2. $\dagger$

Matrix effects. As shown in ESI Table S3, $\uparrow$ the spiked recoveries were $92.5-118.1 \%$ when adding $1000 \mathrm{ng} \mathrm{mL}^{-1}$ of $\mathrm{GSH}$, $2000 \mathrm{ng} \mathrm{mL}{ }^{-1}$ of GSSG, $1000 \mathrm{ng} \mathrm{mL}^{-1}$ of Leu, $6000 \mathrm{ng} \mathrm{mL}^{-1}$ of Trp,

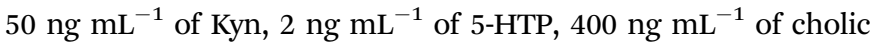
acid, $8 \mathrm{ng} \mathrm{mL}^{-1}$ of 5 -HT, and $2500 \mathrm{ng} \mathrm{mL}^{-1}$ of $N$-phenylacetylglycine into three different plots of rat serum, and when adding $100 \mathrm{ng} \mathrm{mL}{ }^{-1}$ of GSH, $200 \mathrm{ng} \mathrm{mL}{ }^{-1}$ of GSSG, $1000 \mathrm{ng} \mathrm{mL}$ of Leu, $6000 \mathrm{ng} \mathrm{mL} \mathrm{m}^{-1}$ of Trp, $50 \mathrm{ng} \mathrm{\textrm {mL } ^ { - 1 } \text { of Kyn, } 2 \mathrm { ng } \mathrm { mL }} \mathrm{m}^{-1}$ of

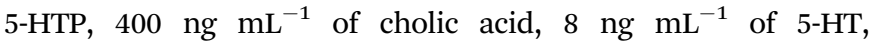
$2500 \mathrm{ng} \mathrm{mL} \mathrm{m}^{-1}$ of $\mathrm{N}$-phenylacetylglycine into three different plots of rat urine with various baseline levels. The CVs of measured Trp and Kyn concentrations from these lots were $\leq 10 \%(n=5)$. These results indicated that different matrixes did not significantly affect the assay.

Analysis of metabolite profiling by pseudo-targeted method. The pseudotargeted method was exploited to investigate the serum and urine metabolite MRM profiling of four groups (blank, diethylstilbestrol, control, and GC).

Firstly, the PCA model was built to exhibit the metabolic distinction of the four groups. From multivariate analysis, there were obvious metabolic differences between the GC groups (including diethylstilbestrol and control groups) and the blank group. The QC samples clustered together tightly in the score plot of PCA, which indicated that the system stability was accommodative for this metabolomics study (Fig. 2).

Then, the critical $P$-value was set to 0.05 for significantly differential metabolites in this research. Accordingly, as shown in Table 2, differential metabolites compared to the blank group were tentatively identified as follows: 17 in serum samples and 
Table 1 Calibration curves of the developed UPLC-MS/MS method

\begin{tabular}{|c|c|c|c|c|c|}
\hline Sample & Compound & Standard curves & $1 / X$ weight & $R^{2}$ & Range \\
\hline \multirow[t]{6}{*}{ Serum } & GSH & $Y=0.0002 X-0.0069$ & $Y=-0.0005 X+0.0001$ & 0.9995 & $100-10000 \mathrm{ng} \mathrm{mL}^{-1}$ \\
\hline & GSSG & $Y=0.0027 X+0.3711$ & $Y=-0.0121 X+0.0023$ & 0.9981 & $200-20000 \mathrm{ng} \mathrm{mL}^{-1}$ \\
\hline & L-Kynurenine & $Y=0.0412 X+0.0153$ & $Y=-0.0049 X+0.0344$ & 0.9980 & $5-500 \mathrm{ng} \mathrm{mL} \mathrm{m}^{-1}$ \\
\hline & L-Tryptophan & $Y=0.0021 X+0.9165$ & $Y=1.6125 X+0.0048$ & 0.9921 & $600-60000 \mathrm{ng} \mathrm{mL}^{-1}$ \\
\hline & 5-HTP & $Y=0.0911 X-0.0099$ & $Y=0.0005 X+0.0883$ & 0.9919 & $0.2-20 \mathrm{ng} \mathrm{mL}^{-1}$ \\
\hline & $N$-Phenylacetylglycine & $Y=0.0162 X+3.1554$ & $Y=-0.3321 X+0.0143$ & 0.9932 & $250-25000 \mathrm{ng} \mathrm{mL}^{-1}$ \\
\hline \multirow[t]{5}{*}{ Urine } & GSH & $Y=0.0031 X-0.1633$ & $Y=-0.0249 X+0.0031$ & 0.9970 & $100-1000 \mathrm{ng} \mathrm{mL}^{-1}$ \\
\hline & GSSG & $Y=0.00002 X+0.0012$ & $Y=-0.00004 X+0.00005$ & 0.9977 & $200-2000 \mathrm{ng} \mathrm{mL} \mathrm{m}^{-1}$ \\
\hline & L-Leucine & $Y=0.0289 X-2.0061$ & $Y=-0.0287 X+0.0295$ & 0.9986 & $100-10000 \mathrm{ng} \mathrm{mL}^{-1}$ \\
\hline & L-Kynurenine & $Y=0.0188 X-0.0315$ & $Y=0.0013 X+0.0182$ & 0.9983 & $5-500 \mathrm{ng} \mathrm{mL}-1$ \\
\hline & L-Tryptophan & $Y=0.0009 X-0.277$ & $Y=-0.0184 X+0.0009$ & 0.9997 & $600-60000 \mathrm{ng} \mathrm{mL}^{-1}$ \\
\hline
\end{tabular}

12 in urine samples for the GC group, 15 in serum samples and 9 in urine samples for the diethylstilbestrol group, 12 in serum samples and 11 in urine samples for the control group, and 11 in serum samples and 7 in urine samples that were simultaneously present in the GC, diethylstilbestrol, and control groups. To further understand the metabolic differences between different groups, a clustering heatmap was generated for all differential metabolites, demonstrating the relative increase (red) or decrease (green) (Fig. 3).

Eighteen differential metabolites simultaneously present in the GC, diethylstilbestrol, and control groups were described as

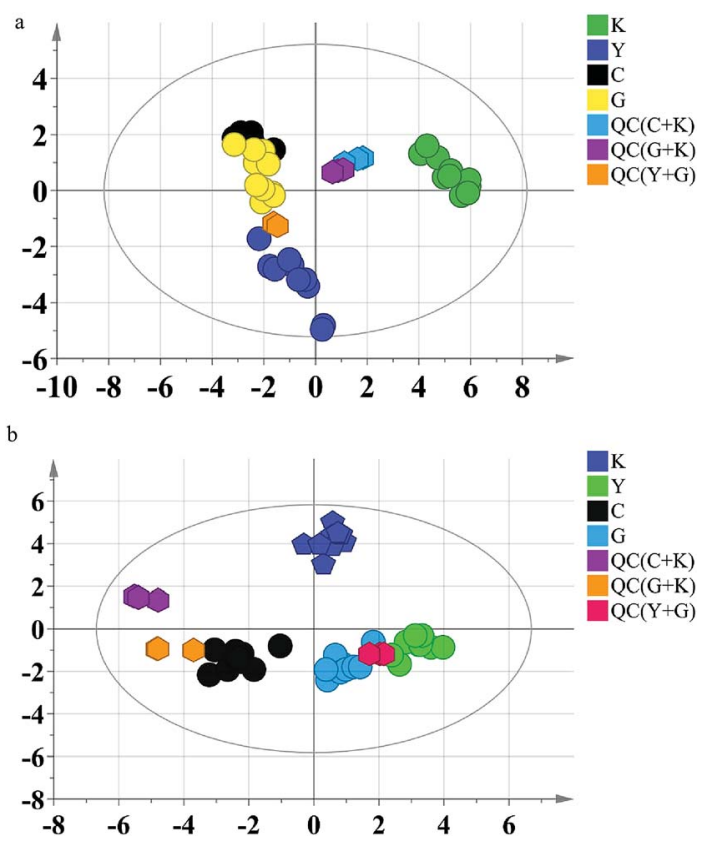

Fig. 2 PCA score plots of rat data. (a) Serum; (b) urine. K: blank group; Y: diethylstilbestrol group; C: control group; G: GC group. follows: glucose, citric acid, proline, betaine, ornithine, $\mathrm{N}$-phenylacetylglycine, phenylpyruvic acid, inosine, C16:0LPC, creatinine, and xanthosine in serum, and benzene acetyl glycine, pyroglutamic acid, acetylcarnitine, and $N_{6}$-acetyl lysine in urine were observed to be significantly decreased $(P<0.05)$; while taurine, ornithine, $\alpha$-ketoglutaric acid, and spermidine in urine were significantly increased $(P<0.05)$. Additionally, taurine (serum) was significantly increased, while $\alpha$-ketoglutaric acid (serum), C18:0LPC (serum), and betaine (urine) were significantly decreased in the diethylstilbestrol and GC groups $(P<$ 0.05); L-kynurenine (serum) was up-regulated in the diethylstilbestrol group, but down-regulated in the GC group $(P<0.05)$; pyroglutamic acid (serum) and proline (urine) were downregulated, and citric acid (urine) and nicotinamide (urine) were markedly increased in control and GC group $(P<0.05)$; and phenylalanine was down-regulated in the GC group $(P<0.05)$.

In this study, the effects of GCs on the uterus of immature rats were investigated. The uterus is the most sensitive organ for assaying the ER-dependent effects of chemicals. Herba Cistanches have been reported to induce an increase in uterine weight by enhancing the lutropin-releasing function of the hypothalamic-pituitary-ovary, ${ }^{20}$ which was also observed for GCs in this study. This indicated that GCs have estrogenic-like activity. Recently, reports have mainly focused on the predominant mechanism by which estrogenic effects are expressed through binding to the ERs, ${ }^{\text {21-23 }}$ but the metabolic mechanism has not been studied in depth. In this study, a pseudometabolomics method was used to explore the estrogenic-like mechanism of GCs.

Metabolic intermediates of a sequential series of reactions changed in a more pronounced fashion than enzymatic kinetics or individual fluxes. ${ }^{24}$ Seventeen metabolites in serum, including glucose, citric acid, taurine, proline, betaine, ornithine, pyroglutamic acid, $\alpha$-ketoglutaric acid, $N$-phenylacetylglycine, phenylpyruvic acid, inosine, C18:0LPC, C16:0LPC, 
Table 2 Differential metabolites in the serum and urine in diethylstilbestrol, control, or GC groups compared to the blank group ${ }^{a}$

\begin{tabular}{|c|c|c|c|c|c|}
\hline Sample & Metabolites & Blank group & Diethylstilbestrol group & Control group & GCs group \\
\hline \multirow[t]{11}{*}{ Serum } & Glucose & $50.54 \pm 5.06^{\# \# \triangle \triangle}$ & $27.91 \pm 4.17^{* *}$ & $33.63 \pm 3.08^{* *}$ & $33.41 \pm 2.42 * *$ \\
\hline & Citric acid & $18.10 \pm 2.49^{\# \# \triangle \triangle}$ & $5.37 \pm 0.70^{* *}$ & $7.16 \pm 0.83^{* *}$ & $6.27 \pm 0.84^{* *}$ \\
\hline & Proline & $62.09 \pm 3.70^{\# \# \triangle \triangle}$ & $27.45 \pm 3.26^{* *}$ & $24.40 \pm 2.83^{* *}$ & $28.32 \pm 2.61^{* *}$ \\
\hline & Betaine & $8.30 \pm 0.58^{\# \# \triangle \triangle}$ & $5.53 \pm 0.67^{* * \Delta} \Delta$ & $2.89 \pm 0.25^{* * \# \#}$ & $3.56 \pm 0.21^{* * \# \#}$ \\
\hline & Ornithine & $0.37 \pm 0.071^{\# \triangle \triangle}$ & $0.24 \pm 0.048^{*}$ & $0.12 \pm 0.016^{* *}$ & $0.14 \pm 0.021^{* *}$ \\
\hline & $\mathrm{N}$-Phenylacetylglycine & $0.87 \pm 0.086^{\# \# \triangle \triangle}$ & $0.35 \pm 0.061^{* *}$ & $0.23 \pm 0.039^{* *}$ & $0.12 \pm 0.019^{* * \# \#}$ \\
\hline & Phenylpyruvic acid & $1.34 \pm 0.10^{\# \triangle}$ & $0.98 \pm 0.12^{*}$ & $1.05 \pm 0.095^{*}$ & $1.02 \pm 0.061^{*}$ \\
\hline & Inosine & $0.96 \pm 0.27^{\# \# \triangle \triangle}$ & $0.11 \pm 0.018^{* *}$ & $0.24 \pm 0.10^{* *}$ & $0.25 \pm 0.055^{* *}$ \\
\hline & C18:0LPC & $1.31 \pm 0.17^{\# \#}$ & $0.70 \pm 0.094^{* * \Delta \triangle} \Delta$ & $1.41 \pm 0.14^{\# \#}$ & $0.82 \pm 0.088^{*} \triangle \triangle$ \\
\hline & C16:0LPC & $2.59 \pm 0.25^{\# \# \triangle \triangle}$ & $1.50 \pm 0.23^{* *}$ & $1.00 \pm 0.15^{* *}$ & $0.98 \pm 0.13^{* *}$ \\
\hline & Creatinine & $0.068 \pm 0.011^{\# \triangle \triangle}$ & $0.042 \pm 0.0066^{*}$ & $0.031 \pm 0.008^{* *}$ & $0.034 \pm 0.0061^{* *}$ \\
\hline & Betaine & $11.33 \pm 2.66^{\#}$ & $5.58 \pm 0.84^{*}$ & $6.63 \pm 1.82$ & $4.65 \pm 0.77^{* *}$ \\
\hline & Taurine & $7.91 \pm 0.84^{\# \# \triangle \triangle}$ & $30.48 \pm 1.85^{* * \Delta \triangle}$ & $17.01 \pm 3.03^{* * \# \#}$ & $44.29 \pm 2.69 * * \# \triangle \Delta \Delta$ \\
\hline & Citric acid & $81.70 \pm 20.08^{\triangle}$ & $116.34 \pm 14.02$ & $152.29 \pm 35.27^{*}$ & $144.44 \pm 12.42^{*}$ \\
\hline & Ornithine & $0.12 \pm 0.018^{\# \Delta \triangle}$ & $0.19 \pm 0.025^{*} \triangle \triangle$ & $0.37 \pm 0.065^{* * \# \#}$ & $0.26 \pm 0.044^{*}$ \\
\hline & Pyroglutamic acid & $32.45 \pm 3.99^{\# \# \triangle \triangle}$ & $13.01 \pm 1.74^{* *}$ & $12.15 \pm 3.30^{* *}$ & $18.05 \pm 2.26^{* *}$ \\
\hline & Acetylcarnitine & $11.25 \pm 1.74^{\# \# \triangle \triangle}$ & $2.91 \pm 0.26^{* *}$ & $4.27 \pm 0.38^{* *}$ & $5.62 \pm 1.06^{* * \#}$ \\
\hline & $N_{6}$-Acetyl lysine & $4.02 \pm 0.41^{\# \# \triangle \triangle}$ & $2.66 \pm 0.30^{* * \Delta \triangle}$ & $1.19 \pm 0.17^{* * \# \#}$ & $2.14 \pm 0.25^{* * \Delta}$ \\
\hline & Nicotinamide & $3.08 \pm 0.72^{\triangle} \triangle$ & $4.06 \pm 0.79^{\triangle}$ & $6.91 \pm 1.01 * * \#$ & $4.79 \pm 0.93^{*}$ \\
\hline & $\alpha$-Ketoglutaric acid & $27.88 \pm 7.52^{\# \Delta \Delta}$ & $50.44 \pm 6.63^{*}$ & $82.30 \pm 17.99^{* *}$ & $52.21 \pm 8.43^{*}$ \\
\hline & Spermidine & $1.29 \pm 0.25^{\# \# \Delta}$ & $6.66 \pm 0.31^{* * \Delta \Delta}$ & $3.57 \pm 0.98^{* \# \#}$ & $8.02 \pm 0.63^{* * \Delta \triangle}$ \\
\hline & Proline & $65.14 \pm 11.54^{\triangle} \triangle$ & $42.26 \pm 10.24^{\triangle}$ & $16.34 \pm 5.23^{* * \#}$ & $31.15 \pm 5.88^{* *}$ \\
\hline
\end{tabular}

${ }^{a}$ Compared to the blank group: ${ }^{*} p<0.05,{ }^{* *} p<0.01$; compared to the diethylstilbestrol group: $\# p<0.05$, \#\#p $<0.01$; compared to the control group: $\Delta p<0.05, \Delta \Delta p<0.01$.

creatinine, phenylalanine, L-kynurenine, and xanthosine, and 12 metabolites in urine, including benzene acetyl glycine, betaine, taurine, citric acid, ornithine, pyroglutamic acid, acetylcarnitine, $N_{6}$-acetyl lysine, nicotinamide, $\alpha$-ketoglutaric acid, spermidine, and proline, were found to be involved in the estrogenic-like mechanism of GCs. Several altered metabolites were of special interest because they were present in both serum and urine. For instance, citric acid, formed by the condensation of acetyl coenzyme A and oxaloacetic acid and playing an important role in the citric acid cycle related to energy metabolism, ${ }^{25}$ was down-regulated in the serum of the GC group, but up-regulated in urine. Furthermore, $\alpha$-ketoglutaric acid with a glutamine carbon frame, which can maintain total nitrogen balance and promote protein synthesis, and is the central material of the citric acid cycle, was down-regulated in the serum of the GC group, but up-regulated in urine. ${ }^{26}$ The citric acid cycle is not only the final metabolic pathway of three major nutrients (carbohydrates, lipids, and amino acids), but also the link among sugar, lipid, and amino acid metabolism, and the main way to obtain energy for the body. ${ }^{27,28}$ This showed that the estrogenic-like mechanism of GCs was related to energy metabolism, which is the same as diethylstilbestrol. Therefore, there was a clear link between the citric acid cycle and the increase in uterine weight in immature rats treated with GCs. As an important methyl donor, betaine plays an important role in fetus growth and development, indicating that betaine is involved in the increase in uterine weight. ${ }^{29}$ Interestingly, taurine was up-regulated in both serum and urine, which could promote lipid digestion and absorption, and cell uptake and utilization of glucose by promoting glucose metabolism. Taurine deficiency has also been reported to lead to weight loss in young animals, suggesting that taurine plays an important role in growth and development. ${ }^{30}$ Moreover, the regulation effect of betaine and taurine by GCs followed the same trend using diethylstilbestrol, but with an enhanced effect compared with diethylstilbestrol.

Furthermore, numerous amino acids were significantly altered. Our results suggested that GCs caused metabolic abnormalities in amino acids. Amino acid metabolism could be used for the synthesis of specific proteins, peptides, and other nitrogenous compounds, or decarboxylation by deamination, transamination, combined with ammonia decomposition, or for energy release through the citric acid cycle. ${ }^{31,32}$ Therefore, alterations of these compounds maybe involved in the important signaling events that trigger the increase in uterine weight.

Moreover, for a detailed pathway analysis, the differential metabolites were categorized into several major pathways including the citrate cycle (TCA cycle), glutathione metabolism, 

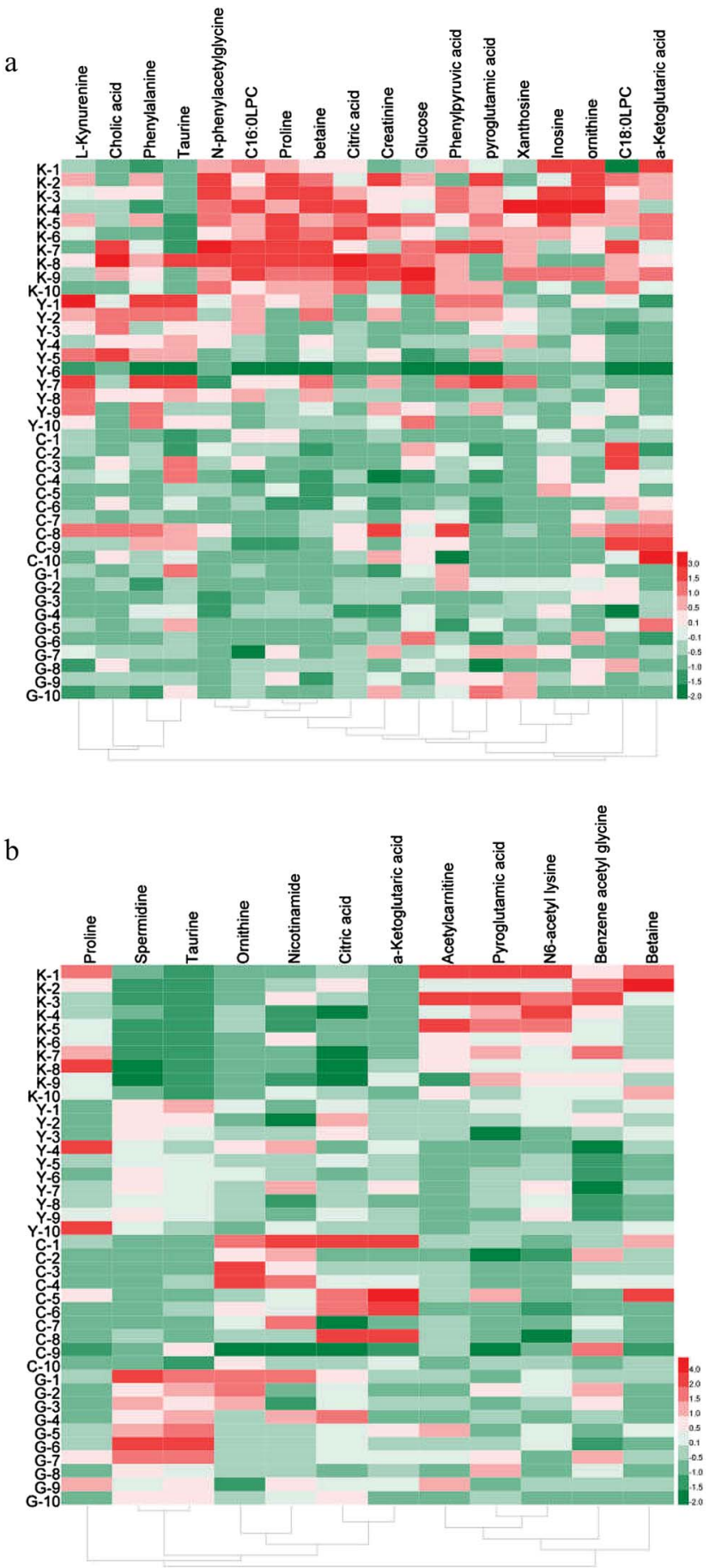

Fig. 3 Heatmap of differential metabolites in different groups. (a) Serum; (b) urine.

arginine and proline metabolism, D-glutamine and D-glutamate metabolism, biosynthesis of phenylalanine, tyrosine and tryptophan, phenylalanine metabolism, and other pathways using Pathway Analysis of MetaboAnalyst software (http://www.metaboanalyst.ca), as shown in Fig. 4. The energy metabolism-related pathway, including the TCA cycle and glutathione metabolism (both in serum and urine), was one of the main targets of estrogenic effects. The critical role of estrogenic chemicals in energy metabolism was verified by ERs
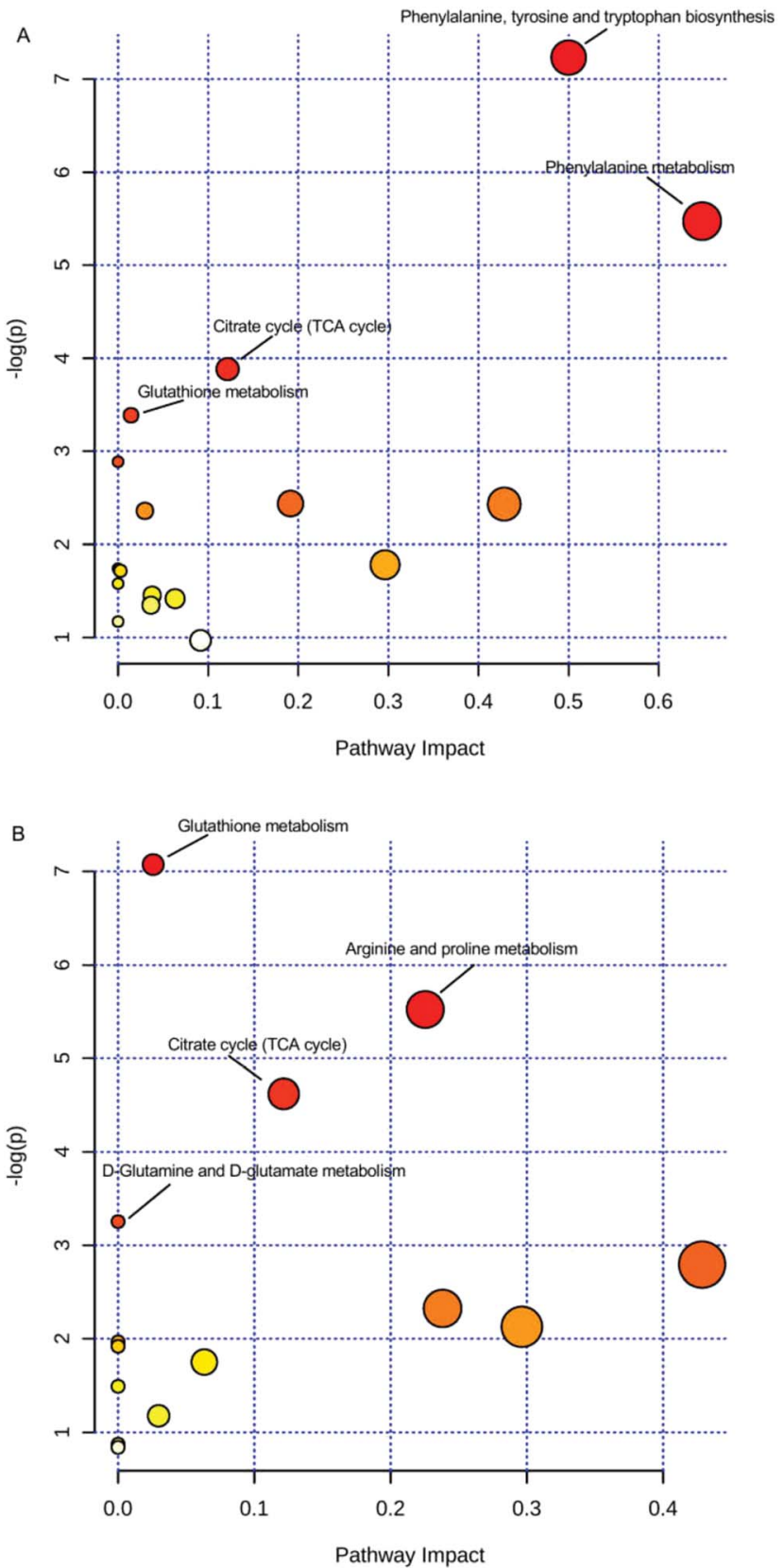

Fig. 4 Pathways of differential metabolites. (A) Serum; (B) urine; small $p$ values and large pathway impact factors indicate that the pathway is greatly influenced.

regulating the genes required for mitochondrial function, TCA cycle, and more, according to previous studies. ${ }^{33,34}$ It could be concluded that the estrogenic-like mechanism of GCs was similar to that of diethylstilbestrol, with both related, to some extent, to the TCA cycle and glutathione metabolism, but with GCs performing better than diethylstilbestrol.

Although the possible mechanisms could not be clarified in this study, some metabolites were selected that could be used to explore other estrogenic mechanisms of GCs in the uterus in the future. Therefore, to better explore the mechanism, other technology, such as proteomics, will be applied in future research. 


\section{Conclusions}

To summarize, a serum and urine pseudotargeted metabolomics method based on UPLC-QTRAP MS was established for exploring the estrogenic-like mechanisms of GCs, which provided robust and reliable results. Using the established pseudotargeted approach, a holistic view of the changes in serum and urine metabolomics of the estrogenic-like mechanism (GCs) was revealed.

\section{Conflicts of interest}

There are no conflicts to declare.

\section{Acknowledgements}

The present study was supported by National Natural Science Foundation of China (81073015) and Harbin Applied Technology Research and Development Project (2015RQQXJ091). Young innovative talent training plan of College in Heilongjiang Province (UNPYSCT-2016182).

\section{References}

1 J. Cano-Nicolau, C. Vaillant, E. Pellegrini, T. D. Charlier, O. Kah and P. Coumailleau, Front. Neurosci., 2016, 10, 112.

2 K. Dumasia, A. Kumar, S. Deshpande and N. H. Balasinor, Epigenetics, 2017, 31, 1-8.

3 G. Mirabolghasemi and Z. Kamyab, Int. J. Fertil. Steril., 2017, 11, 47-55.

4 L. J. Deleruyelle, Int. J. Pharm. Compd., 2016, 20, 447-454.

5 L. J. Deleruyelle, Int. J. Pharm. Compd., 2016, 20, 359-364.

6 T. Usui, Endocr. J., 2006, 53, 7-20.

7 M. D. Shelby, R. R. Newbold, D. B. Tully, K. Chae and V. L. Davis, Environ. Health Perspect., 1996, 104, 1296-1300.

8 L. Gu, W. T. Xiong, C. Wang, H. X. Sun, G. F. Li and X. Liu, Asian J. Androl., 2013, 15, 838-840.

9 F. Peng, R. Xu, X. Wang, C. Xu, T. Liu and J. Chen, Biol. Pharm. Bull., 2016, 39, 2066-2070.

10 T. Wang, X. Zhang, W. Xie and Y. C. Ma, Am. J. Chin. Med., 2012, 40, 1123-1141.

11 Q. Chen, B. Yang, S. Gao and J. J. Zhang, Chin. Herb. Med., 2013, 5, 292-296.

12 W. L. Li, X. M. Sun, H. Song, J. X. Ding, J. Bai and Q. Chen, J. Food Sci., 2015, 80, H2079-H2087.

13 M. N. Samtani and W. J. Jusko, Biomed. Chromatogr., 2007, 21, 585-597.

14 Y. H. Kim, J. M. Kim, J. S. Lee, S. R. Gang, H. S. Lim, M. Kim and O. H. Lee, Food Chem., 2016, 190, 1086-1092.
15 R. Rossi, I. Dalle-Donne, A. Milzani and D. Giustarini, Clin. Chem., 2006, 52, 1406-1414.

16 A. Carretero, Z. León, J. C. García-Cañaveras, A. Zaragoza, M. J. Gómez-Lechón, M. T. Donato and A. Lahoz, Anal. Bioanal. Chem., 2014, 406, 5465-5476.

17 M. Thompson, S. L. R. Ellison and R. Wood, Pure Appl. Chem., 2002, 74, 835-855.

18 Y. Huang, R. Shi, W. Gee and R. Bonderud, Bioanalysis, 2012, 4, 271-279.

19 G. Kaur, E. M. Leslie, H. Tillman, W. M. Lee, D. P. Swanlund and C. J. Karvellas, PLoS One, 2015, 10, e0139299.

20 P. W. Zhao, D. W. Wang, J. Z. Niu, J. F. Wang and L. Q. Wang, Zhongguo Zhongyao Zazhi, 2007, 32, 436-439.

21 S. J. Kim, S. W. Jin, G. H. Lee, Y. A. Kim and H. G. Jeong, Toxicol. Res., 2017, 33, 71-77.

22 F. V. Le, S. Aït-Aïssa, M. Sonavane, J. M. Porcher, P. Balaguer, J. P. Cravedi, D. Zalko and F. Brion, Ecotoxicol. Environ. Saf., 2017, 142, 150-156.

23 S. Zingue, C. B. Nde, T. Michel, D. T. Ndinteh, J. Tchatchou, M. Adamou, X. Fernandez, F. T. Fohouo, C. Clyne and D. Njamen, BMC Complementary Altern. Med., 2017, 17, 65.

24 S. Fernández-Arroyo, A. Gómez-Martínez, L. RocamoraReverte, R. Quirantes-Piné, A. Segura-Carretero, A. Fernández-Gutiérrez and J. A. Ferragut, J. Pharm. Biomed. Anal., 2012, 63, 128-134.

25 P. Lobit, M. Génard, B. H. Wu, P. Soing and R. Habib, J. Exp. Bot., 2003, 54, 2489-2501.

26 E. Riedel, M. Nündel and H. Hampl, Nephron, 1996, 74, 261265.

27 O. E. Owen, S. C. Kalhan and R. W. Hanson, J. Biol. Chem., 2002, 277, 30409-30412.

28 R. S. Dalvi, T. Das, D. Debnath, S. Yengkokpam, K. Baruah, L. R. Tiwari and A. K. Pal, J. Therm. Biol., 2017, 65, 32-40.

29 M. K. Anas, M. A. Hammer, M. Lever, J. A. Stanton and J. M. Baltz, J. Cell. Physiol., 2007, 210, 266-277.

30 J. A. Sturman and J. M. Messing, J. Nutr., 1991, 121, 11951203.

31 J. K. Cleal and R. M. Lewis, J. Neuroendocrinol., 2008, 20, 419426.

32 M. A. Grillo, A. Lanza and S. Colombatto, Amino Acids, 2008, 34, 517-523.

33 C. R. Dufour, M. P. Levasseur, N. H. Pham, L. J. Eichner, B. J. Wilson, A. Charest-Marcotte, D. Duguay, J. F. PoirierHéon, N. Cermakian and V. Giguère, PLoS Genet., 2011, 7, e1002143.

34 C. Chaveroux, L. J. Eichner, C. R. Dufour, A. Shatnawi, A. Khoutorsky, G. Bourque, N. Sonenberg and V. Giguère, Cell Metab., 2013, 17, 586-598. 